\title{
Indian hedgehog couples chondrogenesis to osteogenesis in endochondral bone development
}

\author{
Ung-il Chung, ${ }^{1}$ Ernestina Schipani, ${ }^{1}$ Andrew P. McMahon, ${ }^{2}$ and Henry M. Kronenberg ${ }^{1}$ \\ ${ }^{1}$ Endocrine Unit, Massachusetts General Hospital, Boston, Massachusetts, USA \\ ${ }^{2}$ Department of Molecular and Cellular Biology, Harvard University, Cambridge, Massachusetts, USA
}

Address correspondence to: Henry Kronenberg, Endocrine Unit, Massachusetts General Hospital,

50 Blossom Street, Wellman 503, Boston, Massachusetts 02114, USA.

Phone: (617) 726-3966; Fax: (617) 726-7543; E-mail: kronenberg.henry@mgh.harvard.edu.

Received for publication November 3, 2000, and accepted in revised form December 27, 2000.

\begin{abstract}
Vertebrate skeletogenesis requires a well-coordinated transition from chondrogenesis to osteogenesis. Hypertrophic chondrocytes in the growth plate play a pivotal role in this transition. Parathyroid hormone-related peptide (PTHrP), synthesized in the periarticular growth plate, regulates the site at which hypertrophy occurs. By comparing $\mathrm{PTH} / \mathrm{PTHrP}$ receptor ${ }^{-/} /$wild-type ( $P P R^{-/} /$wild-type) chimeric mice with $\mathrm{Ihb}^{-/-} ; \mathrm{PPR}^{-/-} /$wild-type chimeric and $\mathrm{Ibb}^{-/-} /$wild-type chimeric mice, we provide in vivo evidence that Indian hedgehog (Ihb), synthesized by prehypertrophic and hypertrophic chondrocytes, regulates the site of hypertrophic differentiation by signaling to the periarticular growth plate and also determines the site of bone collar formation in the adjacent perichondrium. By providing crucial local signals from prehypertrophic and hypertrophic chondrocytes to both chondrocytes and preosteoblasts, $I h b$ couples chondrogenesis to osteogenesis in endochondral bone development.
\end{abstract}

J. Clin. Invest. 107:295-304 (2001).

\section{Introduction}

The skeleton of the developing vertebrate limb forms through endochondral bone development. This process is characterized by replacement of a cartilage mold by bone, during which chondrogenesis and osteogenesis are tightly coordinated in time and space $(1,2)$. During chondrogenesis, growth plate chondrocytes proliferate in orderly columns, then stop proliferating and become prehypertrophic chondrocytes. These chondrocytes then hypertrophy, produce a characteristic matrix, and die. Hypertrophic chondrocytes in the growth plate play a pivotal role in coordinating chondrogenesis and osteogenesis, as hypertrophic chondrocytes provide a scaffold for subsequent formation of trabecular bone by mineralizing their surrounding matrix, and they may induce a bone collar, the precursor of cortical bone, in the adjacent perichondrium (3).

The switch from a proliferative to a postproliferative state determines the number of chondrocytes in the proliferative versus hypertrophic pools. Parathyroid hormone-related peptide (PTHrP) is involved in this switch. In the growth plate, PTHrP mRNA is expressed by perichondrial cells and proliferating chondrocytes in the periarticular region, whereas the mRNA for its receptor, the $P T H / P T H r P$ receptor $(P P R)$, is expressed at low levels by proliferating chondrocytes in columns and at higher levels by prehypertrophic chondrocytes (4). Mice homozygous for a null mutation in either the PTHrP gene or the PPR gene show similar abnormalities in the growth plate; proliferation stops prematurely, yielding a short, irregular layer of proliferating chondrocytes (5-7). A similar phenotype has been reported in human fetuses that carry a null mutation in the PPR gene (Blomstrand chondrodysplasia) (8-11). In contrast, increase in $\mathrm{PTHrP}$ signaling produces an opposite phenotype, with the lengthening of columns of proliferating chondrocytes (12-14). Together, these results suggest that $P T H r P$ regulates the switch from a proliferative to a postproliferative state.

Ihh is one of the vertebrate homologs of the Drosophila segment polarity gene, Hedgehog $(H b)(15-17)$. Ihh mRNA is expressed in prehypertrophic and hypertrophic chondrocytes of the mouse embryo (18). Overexpression of Ihh protein through injection of a recombinant retrovirus into embryonic chicken limbs delays hypertrophy of growth plate chondrocytes, as does addition of an active $\mathrm{NH}_{2}$-terminal fragment of Sonic hedgehog (Shh, a relative of Ihh known to mimic Ihh actions) to embryonic mouse limbs in vitro $(6,19)$. These gain-of-function phenotypes are associated with an increase in PTHrP mRNA expression in the perichondrial region at the ends of long bones. In contrast, the Shh fragment has no effect on either $\mathrm{PTHrP}^{-/-}$or $P P R^{-/}$mouse limbs $(6,19)$. Further, mice homozygous for a null-mutation in the $I h b$ gene have no detectable PTHrP mRNA in their growth plate, and hypertrophic chondrocytes predominate in the $I b b^{-/-}$growth plate late in fetal development (20). These data suggest that Ihh delays the switch from proliferation to hypertrophy of chondrocytes by stimulating PTHrP production in the periarticular growth plate. Ihb also stimulates chondrocyte proliferation by a mechanism that is independent of $P$ THrP (21).

Studies of the growth plates of chimeric mice illustrate how chondrocyte proliferation and further differentiation are coordinated. $P P R^{-/-}$ES cells were inject- 
ed into wild-type blastocysts, which then were developed further in foster mothers. In the growth plate of resultant $P P R^{-/} /$wild-type chimeric mice, $P P R^{-/-}$chondrocytes ectopically hypertrophy closer to the articular surface, while the switch from proliferation to hypertrophy of wild-type chondrocytes is delayed, leading to abnormally long columns of wild-type proliferating chondrocytes (3). Ectopically hypertrophied $P P R^{-/-}$ chondrocytes in $P \mathrm{PR}^{-/} /$wild-type chimeric mice produce Ihh much closer to the periarticular growth plate than wild-type cells, and PTHrP mRNA expression in the periarticular growth plate is upregulated. These data suggest that prehypertrophic and hypertrophic chondrocytes may signal their position through Ihh to the periarticular growth plate, which then responds by acting through PTHrP on columnar proliferating chondrocytes to delay their switch to hypertrophy. However, as prehypertrophic and hypertrophic chondrocytes also express other factors such as bone morphogenetic proteins (Bmps) and Wnts, it is still unclear whether Ihh provides the crucial positional signal.

In addition to its actions on chondrocytes, I $h b$ may control osteogenesis as well. Normally, bone collars are formed in the perichondrium abutting prehypertrophic and hypertrophic chondrocytes. In $P P R^{-1-}$ /wild-type chimeric mice, ectopic bone collars are formed in the perichondrium adjacent to ectopically hypertrophied $P P R^{-/-}$chondrocytes (3). I $\mathrm{hb}^{-/-}$mice lack bone collars in their dramatically shortened, deformed limbs (20). When chicken limbs are infected with an Ihh-producing retrovirus, bone collars are induced, despite suppression of hypertrophy of chondrocytes (19). Further, Ihh stimulates osteogenic differentiation of mesenchymal cell lines in vitro, and Shh induces ectopic bone formation in vivo $(22,23)$. These data suggest that Ihh may be involved in bone collar formation. However, it is still unclear whether Ihh, expressed locally in physiological amounts, determines the site of bone collar formation.

We have hypothesized that Ihh synthesized by prehypertrophic and hypertrophic chondrocytes may be responsible both for signaling the relative position of these cells to the periarticular growth plate and for locally signaling the induction of bone collar formation in the adjacent perichondrium. To test this hypothesis in vivo, we have isolated embryonic stem (ES) cell lines homozygous for a null mutation in both the $P P R$ and $I h h$ genes, and generated chimeric mice containing both wild-type and $I h h^{-/-} ; P P R^{-/-}$cells. Comparison of $I \mathrm{hb}^{-1-} ; P P R^{-1-} /$ wild-type chimeras with $P P R^{-/} /$wild-type chimeras has revealed that, in the absence of Ihh, ectopic prehypertrophic/hypertrophic chondrocytes form, but no longer induce ectopic bone collars in the adjacent perichondrium, and their presence no longer induces elongation of the layer of wildtype columnar proliferating chondrocytes nor upregulates PTHrP mRNA expression in the periarticular growth plate. Furthermore, chimeric mice containing both wild-type and $I b h^{-/-}$cells were also generated. In these mice, bone collar formation occurs in the peri- chondrium adjacent to clusters of wild-type prehypertrophic/hypertrophic chondrocytes, whereas bone collar formation does not occur in the perichondrium adjacent to clusters of $\mathrm{Ihb}^{-/-}$prehypertrophic/hypertrophic chondrocytes. These data suggest that Ihh couples cartilage and bone formation by determining the site at which chondrocytes stop proliferating and further differentiate as well as by locally coordinating this process with the differentiation of adjacent osteoblasts.

\section{Methods}

Generation of ES cell lines de novo. To generate ES cell lines homozygous for the $P T H / P T H r P$ receptor-null mutation $\left(P P R^{-1-}\right)$, mice heterozygous for the $P T H / P T H r P$ receptornull mutation $\left(P P R^{+/-}\right)$in a $\mathrm{C} 57 \mathrm{BL} / 6-129 / \mathrm{SvJ}$ background (6) were mated with each other, and blastocysts were collected. One out of 4 of these blastocysts was expected to be $P P R^{-/}$. To generate ES cell lines homozygous for the Ihb-null mutation ( $\left.\mathrm{Ihh}^{-/-}\right)$as well as $P P R^{-/-}$, mice heterozygous for the I $h b$-null mutation $\left(I h b^{+-}\right)$in a 129/SvJ background (20) were mated with $P P R^{+/-}$mice in a C57BL6-129/SvJ background. Among pups, $I h h^{+/-} ; P P R^{+/-}$mice were selected by Southern blot analysis. Then, these $I h b^{+/-} ; P P R^{+/-}$mice were mated with each other, and blastocysts were collected. One of 16 of these

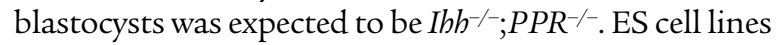
were established from blastocysts as described previously (24). The animal care was in accordance with the policies of the Massachusetts General Hospital.

Generation of chimeric mice. Chimeras were generated by blastocyst injection as described previously (25). $P P R^{-/-}$, $\mathrm{Ibh}^{-1-} ; \mathrm{PPR}^{-1-}$, and $\mathrm{Ibh}^{-{ }^{-}}$ES cells were injected into C57BL/ 6 blastocysts that carry a $\beta$-galactosidase transgene as a marker. To introduce a $\beta$-galactosidase transgene into host blastocysts, C57BL/6 male mice carrying a $\beta$-galactosidase transgene that was engineered to be expressed ubiquitously (26) were mated with wild-type C57BL/6 female mice. One of two blastocysts was expected to carry the transgene. To produce chimeras with various degrees of ES cell contributions, the number of ES cells injected into the blastocele cavity was varied from five to 15. At least two independently established ES cell lines of each genotype yielded an identical phenotype. All the ES cell lines gave germline transmission. Resultant chimeric mice were studied at E14.5, 15.5, 16.5, 17.5, and 18.5 , and mostly the data from d17.5 embryos were presented. All observations in the results were verified in at least five different chimeric mice. The degree of chimerism was estimated by staining for $\beta$-galactosidase activity of the skin and the periarticular growth plate. In $I b b^{-1-} ; P P R^{-1-}$ wild-type chimeric (and also $\mathrm{Ibh}^{-/}$/ wild-type chimeric) mice, the entire length of bone starts becoming shorter than that of wild-type littermates as the degree of contributions from mutant cells goes over approximately $50 \%$. Therefore, when the length of the layer of columnar proliferating chondrocytes in $I b h^{-1-} ; P P R^{-1-}$ /wild-type chimeric mice was compared with that of wild-type littermates, chimeric mice with less than $50 \%$ contributions from mutant cells were used. 
Southern blot analysis. Southern blot analysis was performed as described previously (20). To detect disrupted alleles of the PPR gene, $10 \mu \mathrm{l}(\sim 10 \mu \mathrm{g})$ of genomic DNA were digested with SacI, Southern blotted, and probed with a $1.0-\mathrm{kb}$ SacI-KpnI fragment containing a part of the first intron. The sizes of genomic DNA fragments expected from the normal and disrupted alleles are 5.6 and $4.9 \mathrm{~kb}$, respectively. To detect disrupted alleles of the Ihb gene, $10 \mu \mathrm{l}(\sim 10 \mu \mathrm{g})$ of genomic DNA were digested with NcoI, Southern blotted, and probed with a 1.2-kb KpnI-HindIII fragment containing a part of the 5 -end of the gene. The sizes of genomic DNA fragments expected from the normal and disrupted alleles are 7.6 and $8.4 \mathrm{~kb}$, respectively. To detect $\beta$-galactosidase transgene alleles, $10 \mu \mathrm{l}(\sim 10 \mu \mathrm{g})$ of genomic DNA were digested with StuI, Southern blotted, and probed with a 140-bp fragment containing a 5 '-flanking region of the transgene (26). The sizes of DNA fragments expected from the normal and the mutant alleles are $\sim 7$ and $\sim 12 \mathrm{~kb}$, respectively.

Histological analysis. Chimeras were sacrificed at various ages, dissected, and fixed in 4\% paraformaldehyde/PBS at $4^{\circ} \mathrm{C}$ for 4 hours. For detection of $\beta$-galactosidase activity, tissues were stained with X-Gal (5-bromo-4-chloro3 -indolyl $\beta$-D-galactoside) as described previously (27). Subsequently, they were processed, embedded in paraffin, and cut. Sections were stained with hematoxylin and eosin (H\&E) or Nuclear Fast Red (Fluka, Neu-Ulm, Switzerland) for morphological study. For detection of mineralization, sections were stained with $1 \%$ silver nitrate (von Kossa method); mineral is stained black.

Analysis of $\mathrm{BrdU}$ incorporation. Pregnant mice were injected intraperitoneally with $50 \mu \mathrm{g}$ BrdU per gram body weight 1 hour before sacrifice. Embryos were treated to generate sections as already described here. BrdU was detected by BrdU Staining Kit (Zymed Laboratories Inc., South San Francisco, California, USA) according to the manufacturer's instruction. All the BrdU-positive and -negative nuclei of columnar proliferating chondrocytes were counted. At least three sections were counted for each of two wild-type and two $P P R^{-/-}$/wild-type chimeric littermate embryos at E17.5.

In situ bybridization. Tissues were fixed in $4 \%$ paraformaldehyde/PBS overnight at $4{ }^{\circ} \mathrm{C}$, processed, embedded in paraffin, and cut. In situ hybridization was performed as described previously (4) by using complementary ${ }^{35} \mathrm{~S}$-labeled riboprobes for mouse type $X$ collagen (gift from B. Olsen, Harvard Medical School, Boston, Massachusetts, USA), mouse Ptc1 (R. Johnson, Stanford University, Stanford, California, USA), mouse Ihb (17), mouse PTHrP (J. Wysolmerski, Yale University, New Haven, Connecticut, USA), mouse Id1 (R. Benezra, Memorial Sloan-Kettering, New York, New York, USA), mouse osteocalcin, mouse Cbfa1 (G. Karsenty, Baylor College of Medicine, Houston, Texas, USA), mouse Bmp2 through 7 (E. Robertson, Harvard University, Cambridge, Massachusetts, USA), human Follistatin (Y. Sidis and H. Keutmann, Massachusetts General Hospital, Boston, Massachusetts, USA), mouse
Noggin (R. Harland, University of California at Berkeley, Berkeley, California, USA), mouse Gremlin (A. Zuniga, University of Utrecht, Utrecht, The Netherlands), mouse Dan (ICRFc100D11145; Resource Center of the German Human Genome Project at the Max-PlanckInstitute for Molecular Genetics, Berlin, Germany), and mouse Chordin (S. Ang, IGBMC, Strasbourg, France).

Generation of transgenic mice. Mice expressing a constitutively active human $P P R$ transgene under the control of the rat $\alpha 1$ (II) collagen promoter (caPPR) were generated and identified, as described previously (13). To evaluate chondrocyte-specific expression of the transgene, RTPCR was performed as described previously (13). In brief, total RNA was extracted from sternal cartilage and from calvariae of newborn transgenic mice, and reverse-transcribed into a single-stranded DNA with a reverse primer G2, specific to the human PPR gene. PCR was then performed using the same reverse primer G2 and the forward primer $2 \mathrm{~F}$. The PCR products were analyzed by Southern blot using the ${ }^{32}$ P-labeled oligonucleotides $\mathrm{H} 40$ and 2FA, specific to the human PPR gene. Chondrocytespecific expression of the transgene was also confirmed by in situ hybridization with an antisense probe for the $\mathrm{NH}_{2}$-terminal extracellular domain portion of the human $P P R$ gene, which is significantly different from that of the mouse $P P R$ gene in nucleotide sequence and does not recognize the mouse PPR mRNA (28).

To rescue $P T H r P^{-1-}$ mice, the $c a P P R$ transgene was introduced into $\mathrm{PTHr} \mathrm{P}^{+/-}$mice by appropriate matings, and these mice were mated together to generate caPPR $^{+} ; \mathrm{PTHrP}^{-/-}$mice.

\section{Results}

Isolation of $\mathrm{Ihb}^{-/-} ; \mathrm{PPR}^{-/-}$ES cells and generation of chimeric mice. ES cell lines homozygous for a null-mutation in both the Ibh and PTH/PTHrP receptor genes were generated de novo from inner cell masses of blastocysts derived from mating $I h b^{+/-} ; P P R^{+/-}$mice with each other. Of 20 ES cell lines established, two double homozygous mutants were isolated. In addition, ES cell lines with other genetic combinations such as wild-type, $\mathrm{Ib} \mathrm{h}^{+/-}$, $\mathrm{Ihb}^{-/-}, \mathrm{PPR}^{+/-}$, and $\mathrm{PPR}^{-/-}$cell lines were also isolated. To distinguish visually between cells descended from injected ES cells and cells descended from host blastocysts, one copy of a $\beta$-galactosidase transgene engineered to be expressed widely in mouse tissues (26) was introduced into host blastocysts by appropriate matings. All cells in the growth plate from the mice carrying one copy of the $\beta$-galactosidase transgene alone exhibited $\beta$-galactosidase activity and normal morphology (data not shown). In mice generated by injecting wildtype ES cells into wild-type blastocysts expressing one copy of the $\beta$-galactosidase transgene, cells derived from ES cells and cells derived from host blastocysts behaved indistinguishably, as expected (data not shown).

$\mathrm{Ibh}^{-1-} ; \mathrm{PPR}^{-/-}$chondrocytes undergo ectopic bypertrophy in the absence of ectopic Ihb signaling. The wild-type fetal growth plate consists of three major layers of chondrocytes; that is, the proliferating, prehypertrophic, and 


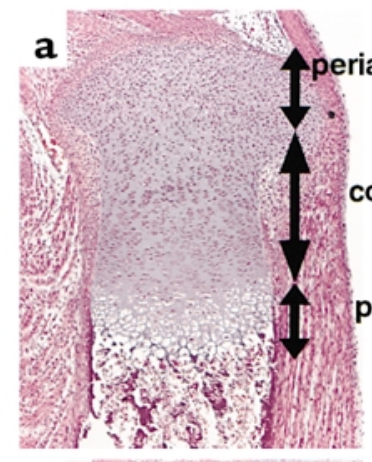

\section{icular proliferating chondrocytes}

columnar proliferating chondrocytes

\section{prehypertrophic \& hypertrophic chondrocytes}

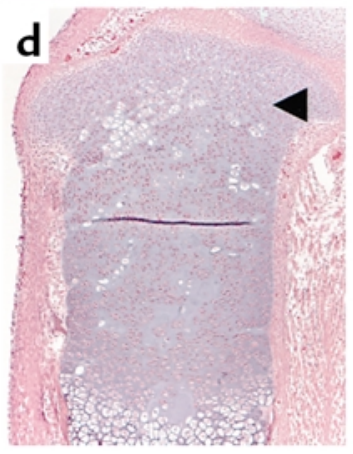

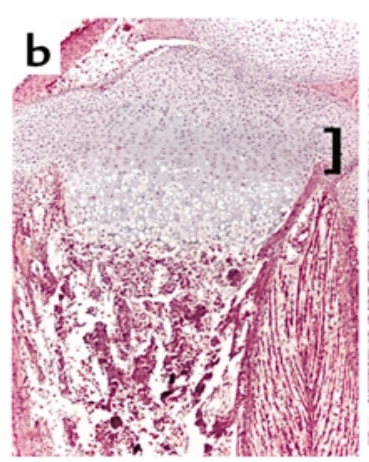

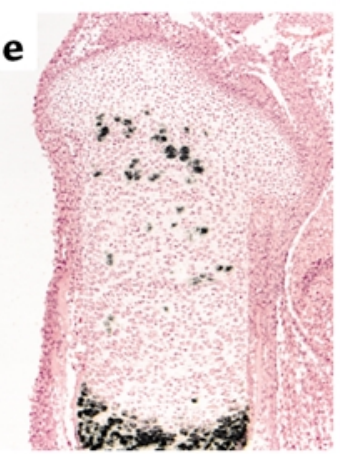

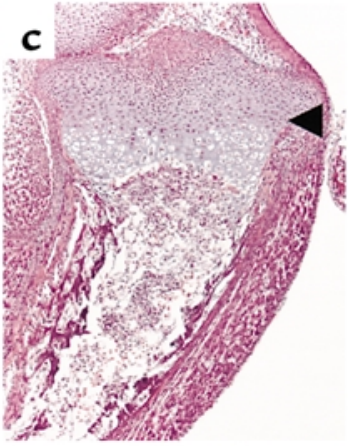

g

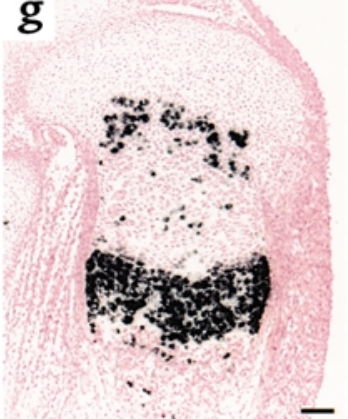

\section{Figure 1}

Ectopic differentiation of growth plate chondrocytes deficient in PTHrP signaling. (a-c) Sections of the tibiae from d17.5 wild-type (a), $P T H r P^{-/-}(\mathbf{b})$, and $P P R^{-/-}(\mathbf{c})$ embryos were stained with H\&E. The growth plates of $P T H r P^{-/-}$mice and $P P R^{-/-}$mice have a shorter layer of columnar proliferating chondrocytes. In $P T H r P^{-1-}$ mice, they represent less than $30 \%$ of wild-type (b, bracket), and in $P P R^{-1-}$ mice, they are almost completely absent (c, arrowhead). ( $\mathbf{d}-\mathbf{g}) \mathrm{H} \& \mathrm{E}$ staining and in situ hybridization with a mouse type $X$ collagen antisense probe of the sections of the tibiae from d17.5 $P P R^{-/-} /$wild-type chimera ( $\mathbf{d}$ and $\mathbf{e}$, respectively) and $/ h h^{-/-} ; P P R^{-/-} /$wild-type chimera (f and $\mathbf{g}$, respectively) embryos. In the absence of $P T H r P$ signaling, mutant cells ectopically hypertrophy when they move from the layer of periarticular proliferating chondrocytes into the layer of columnar proliferating chondrocytes (arrowheads). Staining for $\beta$-galactosidase activity of both chimeric growth plates shows that all the mutant chondrocytes undergo ectopic hypertrophy, whereas wild-type cells do not (data not shown). Horizontal bar $=100 \mu \mathrm{m}$.

hypertrophic layers. The proliferating layer contains two distinct types of chondrocytes. Proliferating chondrocytes close to the articular surface are round, randomly scattered (Figure 1a), and express PTHrP mRNA. We refer to this population as periarticular proliferating chondrocytes. Proliferating chondrocytes immediately below periarticular proliferating chondrocytes are flat, form longitudinal columns (Figure 1a), and weakly express PPR mRNA. We refer to this population as columnar proliferating chondrocytes. As previously shown, the growth plates of $\mathrm{PTHr}^{-/-}$mice and $\mathrm{PPR}^{-/-}$ mice have a shortened layer of proliferating chondrocytes (Figure 1, b and c). Columnar proliferating chondrocytes are mainly affected by loss of $P T H r P$ signaling (in $\mathrm{PTHrP}^{-/-}$mice, they represent less than $30 \%$ of wildtype, and in $\mathrm{PPR}^{-/-}$mice, they are almost completely absent), whereas the periarticular proliferating chondrocytes appear relatively intact (Figure 1, b and c). In the growth plate of chimeric mice containing $P P R^{-/-}$ and wild-type cells, $P P R^{-/-}$chondrocytes ectopically adopt the hypertrophic phenotype, as they progress from the layer of periarticular proliferating chondrocytes to the layer of columnar proliferating chondrocytes (Figure 1, $\mathrm{d}$ and e). Thus, PTHrP signaling directly prevents the switch from proliferation to hypertrophy of columnar proliferating chondrocytes. In the growth plate of chimeric mice containing $\mathrm{Ihb}^{-1-} ; P P R^{--}$and wildtype cells, mutant chondrocytes similarly hypertrophy ectopically in the layer of columnar proliferating chondrocytes (Figure 1, fand g). Staining for $\beta$-galactosidase activity of both chimeric growth plates shows that all the mutant chondrocytes undergo ectopic hypertrophy, whereas wild-type cells do not (3) (data not shown).

Ihh signals the relative position of prebypertrophic and hypertrophic chondrocytes to the periarticular growth plate. Previous studies have suggested that prehypertrophic and hypertrophic chondrocytes may signal their position to the periarticular growth plate, which then delays the switch from proliferation to hypertrophy of columnar proliferating chondrocytes through $P T H r P$ signaling. As a result, a certain distance between the periarticular growth plate and the layers of prehypertrophic and hypertrophic chondrocytes is maintained. This distance affects bone length by determining the number of chondrocytes in proliferative versus hypertrophic pools, as hypertrophic chondrocytes contribute to longitudinal bone growth and proliferating chondrocytes serve as a reservoir for future hypertrophic chondrocytes. Ihh may signal the relative position of prehypertrophic and hypertrophic chondrocytes to the periar- 
ticular growth plate (3). To test this hypothesis in vivo, we have compared $P P R^{-1-} /$ wild-type chimeric mice and $I h^{-1-} ; P P R^{-1-} /$ wild-type chimeric mice.

In the wild-type growth plate, Ihh mRNA is expressed in prehypertrophic and hypertrophic chondrocytes (18) (Figure 2a). In $P P R^{-/-} /$wild-type chimeric mice, mutant ectopic prehypertrophic/hypertrophic chondrocytes also express Ihh mRNA (Figure $2 \mathrm{~b}$, asterisk), whereas in $\mathrm{Ihb}^{-1-} ; \mathrm{PPR}^{-/-}$/wild-type chimeric mice, mutant prehypertrophic/hypertrophic chondrocytes do not express Ihh mRNA, as expected (Figure 2c). Patched1 (Ptc1) is a receptor for Hedgehog (Hh) proteins $(29,30)$. Because its transcription is upregulated by $H h$ signaling, Ptc $1 \mathrm{mRNA}$ expression is a marker for actions of Hh proteins (31). In the wild-type growth plate, Ptc1 mRNA is expressed most strongly in columnar proliferating chondrocytes close to Ihh-expressing prehypertrophic/hypertrophic chondrocytes, with its expression decreasing toward the end of bone (3) (Figure $2 \mathrm{~d}$ ). In the growth plate of $P P R^{-/} /$wild-type chimeric mice, however, proliferating chondrocytes surrounding ectopic prehypertrophic/hypertrophic chondrocytes overexpress Ptc1 mRNA, providing evidence for ectopic action of Ihh (Figure 2e, bracket). In contrast, in the growth plate of $I h h^{-/-} ; P P R^{-1-} /$ wild-type chimeric mice, there is no overexpression of Ptc1 mRNA in proliferating chondrocytes surrounding ectopic prehypertrophic/hypertrophic chondrocytes, and the expression pattern of Ptc1 mRNA looks similar to that in the wild-type growth plate, except that mutant ectopic hypertrophic chondrocytes do not express Ptc1 mRNA (Figure 2f). Thus, although
$I b^{-1-} ; P P R^{-1-}$ chondrocytes do ectopically hypertrophy, they do not induce the I $h b$ signaling pathway ectopically, unlike $P P R^{-/-}$chondrocytes.

The growth plate of $P P R^{--} /$wild-type chimeric mice exhibits elongation of the layer of wild-type columnar proliferating chondrocytes, compared with the wildtype growth plate (3) (Figure 3, a and b). The proliferation rate of columnar proliferating chondrocytes of the $P P R^{-1-} /$ wild-type chimeric growth plate is not significantly different from that of columnar proliferating chondrocytes of the wild-type growth plate (percentage of BrdU-positive nuclei: $11.0 \pm 0.5 \%$ vs. $11.8 \pm 0.6 \%$, respectively). Given that Ihh is ectopically expressed, this ectopic Ihh signaling may upregulate PTHrP expression, which, in turn, might delay hypertrophic differentiation of wild-type columnar proliferating chondrocytes. In the growth plate of $\mathrm{Ihb}^{-/-} ; \mathrm{PPR}^{-1-} /$ wildtype chimeric mice, the length of the layer of wild-type columnar proliferating chondrocytes remains the same as the length of the layer of columnar proliferating chondrocytes in the wild-type growth plate, despite the presence of ectopic prehypertrophic/hypertrophic chondrocytes (Figure 3, c and d). Thus, ectopically synthesized Ihh is required to trigger the lengthening of columns of wild-type proliferating chondrocytes.

In the wild-type growth plate, PTHrP mRNA is expressed weakly in periarticular proliferating chondrocytes and adjacent perichondrial cells at E17.5 day (Figure 3e). If the position of prehypertrophic and hypertrophic chondrocytes is sensed by the periarticular growth plate, which then upregulates PTHrP mRNA expression to slow down the switch from proliferation

\section{Figure 2}

Ih signaling by ectopically hypertrophied $P P R^{-/-}$and Ihh ${ }^{-1-} ; P P R^{-1-}$ chondrocytes. (a-c) In situ hybridization of the sections of the tibiae from $\mathbf{d} 17.5$ embryos with a mouse Ihh antisense probe. In the wild-type growth plate (a), thh mRNA is expressed in prehypertrophic and hypertrophic chondrocytes. In the chimeric growth plates, ectopically differentiated $P P R^{-/-}$chondrocytes also express Ihh mRNA (b, asterisk), whereas ectopically differentiated $I h h^{-/-} ; P P R^{-/-}$cells do not (c). (d-f) In situ hybridization of the sections of the tibiae from $\mathrm{d} 17.5$ embryos with a mouse Ptc1 antisense probe. Ptc1 is a transcriptional target of Ihh signaling. In the wild-type growth plate (d), Ptc1 mRNA is expressed most strongly in columnar proliferating chondrocytes adjacent to prehypertrophic chondrocytes with the expression decreasing toward the end of bone. Ptc1 mRNA is also expressed in the perichondrium and the primary spongiosa. In the $P P R^{-1-}$ /wild-type chimeric growth plate (e), periarticular and columnar proliferating chondrocytes surrounding ectopic prehypertrophic/hypertrophic chondrocytes overexpress Ptc1 mRNA (bracket), reflecting ectopic actions of Ihh. In contrast, in the $I h h^{-1-} ; P P R^{-/-} /$wild-type chimeric growth plate $(\mathbf{f})$, there is no ectopic expression of Ptc1 mRNA despite the presence of ectopic prehypertrophic/hypertrophic chondrocytes. Left, bright field; right, dark field. Horizontal bar $=100 \mu \mathrm{m}$.
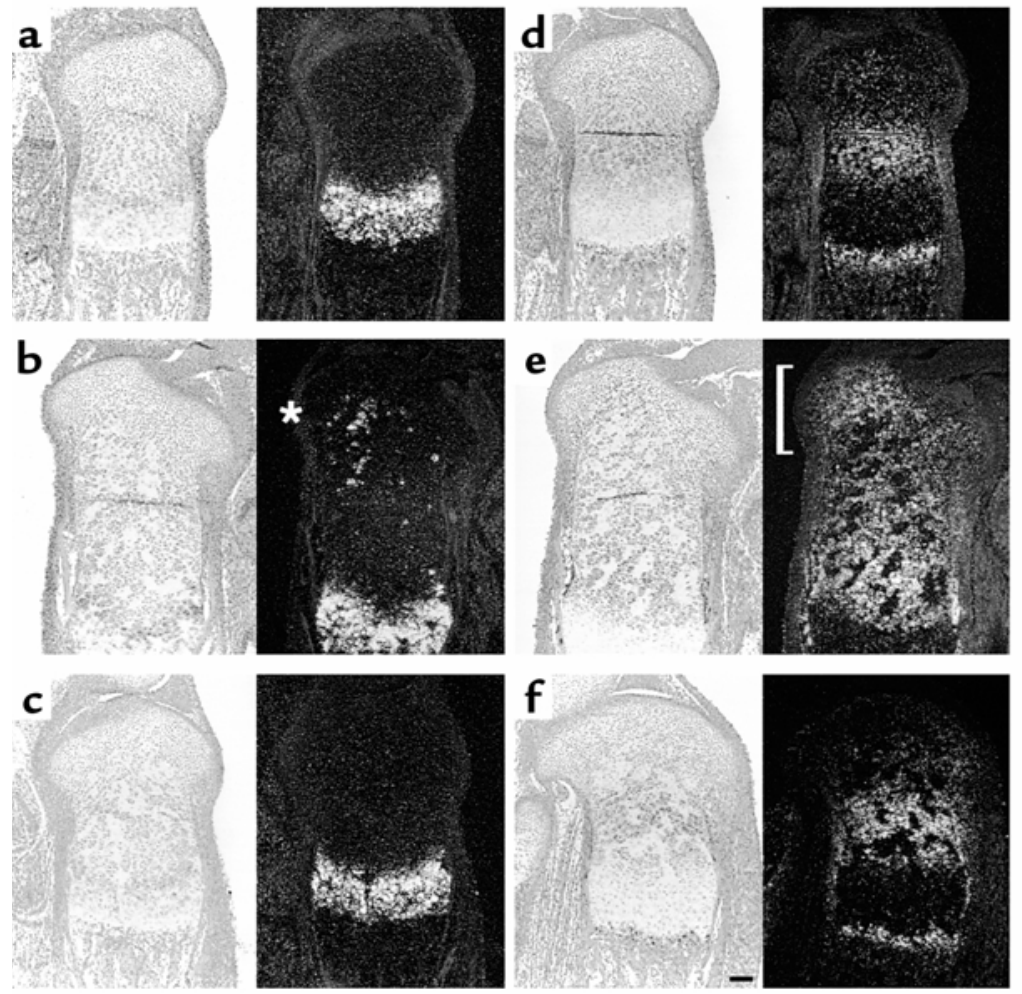


\section{Figure 3}

Ihh signals the position of prehypertrophic and hypertrophic chondrocytes. (a-d) H\&E staining of sections of the tibiae from $\mathrm{d} 17.5$ wild-type (a), $P P R^{-1-} /$ wild-type chimera (b), and $I h h^{-1-} ; P P R^{-1-} /$ wild-type chimera (c and d) embryos. The presence of ectopic hypertrophic chondrocytes in the $P P R^{-/-}$/wildtype chimeric growth plate induces elongation of the layer of wild-type columnar proliferating chondrocytes, leading to greater distance between the layers of periarticular proliferating chondrocytes and prehypertrophic/hypertrophic chondrocytes (compare $\mathbf{a}$ and $\mathbf{b}$ ). In the Ihh-/-;PPR ${ }^{-1-} /$ wild-type chimeric growth plate, however, the presence of ectopic hypertrophic chondrocytes does not induce elongation of the layer of wild-type columnar proliferating chondrocytes ( $\mathbf{c}$ and $\mathbf{d}$, in increasing order of chimerism). Perpendicular bars indicate the length of the layer of columnar proliferating chondrocytes. (e-h) In situ hybridization of sections of the tibiae from d17.5 embryos with a mouse PTHrP antisense probe. In the wild-type growth plate (e), PTHrP mRNA is weakly expressed in the periarticular proliferating chondrocytes, whereas in the $P P R^{-1-}$ growth plate $(\mathbf{f})$, its expression is strongly upregulated in the same area (bracket). In the $P P R^{-/-} /$wildtype chimeric growth plate $(\mathbf{g})$, PTHrP mRNA expression is upregulated in the periarticular proliferating chondrocytes, but to a lesser extent compared with the $P P R^{-/}$growth plate (bracket). In contrast, despite the presence of ectopic hypertrophic chondrocytes, there is no upregulation of PTHrP mRNA expression in the $I h h^{-/-} ; P P R^{-/-}$/ wild-type chimeric growth plate (h). PTHrP mRNA expression is more clearly seen in sections of the wild-type and $I h h^{-/-} ; P P R^{-/-} /$wild-type chimeric growth plates exposed twice as long (data not shown); the sections shown in $\mathbf{e}$ and $\mathbf{h}$, however, were exposed for the same time as those in $\mathbf{f}$ and $\mathbf{g}$ to allow direct comparison. Left, bright field; right, dark field. Horizontal bar $=100 \mu \mathrm{m}$.
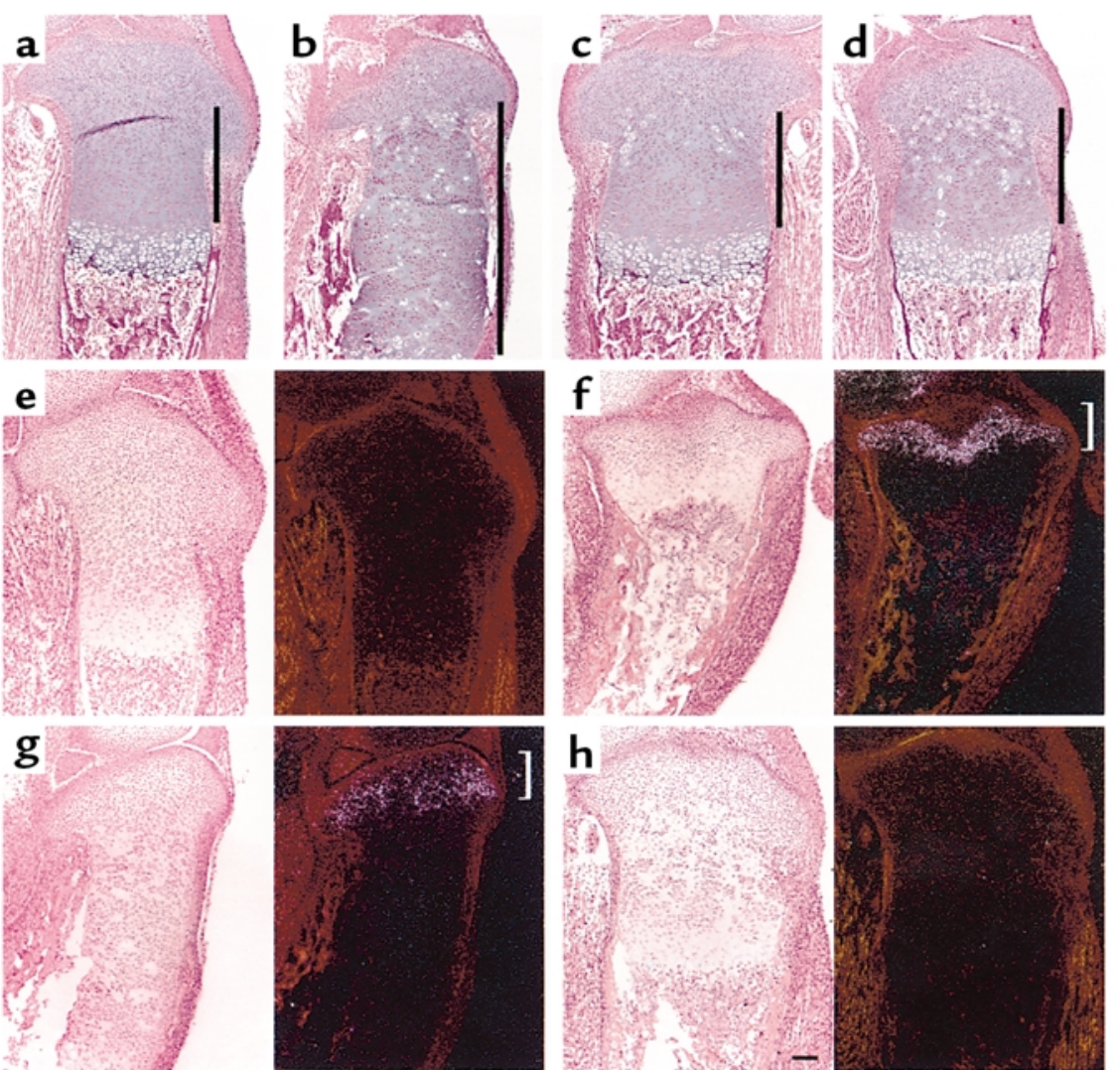

to hypertrophy of columnar proliferating chondrocytes, PTHrP mRNA expression is expected to increase when prehypertrophic and hypertrophic chondrocytes are placed closer than normal to the periarticular growth plate. In the growth plate of $P P R^{-/-}$mice, there are almost no columnar proliferating chondrocytes, and therefore the layer of periarticular proliferating chondrocytes is placed immediately next to the layers of prehypertrophic and hypertrophic chondrocytes. Consistent with the hypothesis just described, PTHrP mRNA expression is strongly upregulated in the $P R^{-/-}$periarticular growth plate (Figure $3 \mathrm{f}$, bracket). In the growth plate of $P P R^{-1-} /$ wild-type chimeric mice, ectopic prehypertrophic/hypertrophic chondrocytes are also placed next to the layer of periarticular proliferating chondrocytes, but fewer in number compared to the $\mathrm{PPR}^{-/}$ growth plate. Therefore, PTHrP mRNA expression is expected to increase, but to a lesser extent than in the $\mathrm{PPR}^{-/}$growth plate. PTHrP mRNA in the growth plate of $P P R^{-1-}$ /wild-type chimeric mice confirms this expectation (Figure $3 \mathrm{~g}$, bracket). In contrast, in the growth plate of $I h h^{-1-} ; P P R^{-1-} /$ wild-type chimeric mice, the presence of ectopic prehypertrophic/hypertrophic chondrocytes does not lead to upregulation of PTHrP mRNA expression in the periarticular growth plate (Figure $3 \mathrm{~h}$ ).
Taken together, these data strongly suggest that Ihh, synthesized by prehypertrophic and hypertrophic chondrocytes, signals the relative position of these cells to the periarticular growth plate, which then responds by upregulating PTHrP mRNA expression to delay the switch to hypertrophy of columnar proliferating chondrocytes.

Ihh determines the location of bone collar formation. Bone collars are precursors of the cortical regions of long bones. Normally, bone collars form in the perichondrium abutting prehypertrophic and hypertrophic chondrocytes (Figure 4a, arrowhead). Bone collars in $\mathrm{PTHrP}^{-/-}$mice and $P P R^{-/-}$mice are also formed in the perichondrium abutting prehypertrophic and hypertrophic chondrocytes, despite much shorter layers of columnar proliferating chondrocytes $(5,6)$. As reported previously (3), in the growth plate of $P \mathrm{PR}^{-1-} /$ wild-type chimeric mice, ectopic bone collars are formed near clusters of ectopic prehypertrophic/hypertrophic chondrocytes (Figure 4b, brackets). These ectopic bone collars express typical markers for osteoblasts, such as type I collagen, alkaline phosphatase, Cbfa1, bone sialoprotein (data not shown), osteopontin (Figure 5b, bracket), and osteocalcin (Figure $5 e$, bracket) mRNAs. These results suggest that hypertrophy of chondrocytes and differentiation of osteoblasts are coupled. To demonstrate that prehypertrophic and 
hypertrophic chondrocytes induce bone collar formation, we took advantage of the rescue of the $\mathrm{PTHrP}^{-/-}$mice by a constitutively active PPR (caPPR) transgene (13). Ribs, with long arrays of hypertrophic chondrocytes and associated bone collar, were examined to determine the effect of manipulating hypertrophic differentiation of chondrocytes. Expression of type X collagen mRNA (Figure 4, $\mathrm{e}-\mathrm{g}$ ), and mineralization (Figure $4, \mathrm{~h}-\mathrm{j}$ ) were analyzed. Normally, rib cartilage close to the sternum does not hypertrophy prenatally, and no bone collar forms (Figure 4 , e and h). In $\mathrm{PTHrP}^{-/-}$mice, however, chondrocytes in the same region prematurely hypertrophy and at the same time acquire bone collars in the adjacent perichondrium (Figure 4, $\mathrm{f}$ and i). When a caPPR gene, which is engineered to be expressed only in chondrocytes under the control of type II collagen promoter (13), is introduced by appropriate matings into $\mathrm{PTHrP} \mathrm{P}^{-/-}$mice, ectopic hypertrophy of rib cartilage is reversed, in association with the disappearance of ectopic bone collars (Figure 4, $\mathrm{g}$ and $\mathrm{j}$ ). Similar findings are observed in the tibia (data not shown). Because the transgene is expressed in chondrocytes, but not in osteoblasts (as assessed by PCR in these mice, data not shown), these data strongly suggest that prehypertrophic and hypertrophic chondrocytes induce bone collars in the adjacent perichondrium.

As noted earlier here, Ihh may provide the local signal whereby prehypertrophic and hypertrophic chondrocytes induce bone collar formation. In the growth plate of $\mathrm{Ihb}^{-1-} ; P P R^{-1-} /$ wild-type chimeric mice, although mutant cells ectopically hypertrophy, they fail to induce ectopic bone collars in the adjacent perichondrium (Figure 4, c and d), and no ectopic expression of osteopontin and osteocalcin mRNAs was observed in this region (Figure 5, $\mathrm{c}$ and f). Furthermore, chimeric mice containing wild-type and $\mathrm{Ibh}^{-/-}$cells were generated. In the growth plate of $\mathrm{Ihb}^{-/-} /$wild-type chimeric mice, mutant cells do not ectopically hypertrophy, but instead hypertrophy in synchrony with wild-type cells. The architecture of the growth plate of $\mathrm{Ihb}^{-1-}$-wild-type chimeric mice is well preserved despite its smaller size, probably due to Ihh secret-

\section{Figure 4}

Ihh determines the location of bone collar formation. (a-d) Sections of the tibiae from $\mathrm{d} 17.5$ embryos were stained by von Kossa method and counterstained with Nuclear Fast Red. Mineral is stained black; nuclei are stained red. In the wild-type growth plate (a), bone collars are formed in the perichondrium adjacent to the layers of prehypertrophic and hypertrophic chondrocytes, whereas in the $P P R^{-/-} /$wild-type chimeric growth plate (b), ectopic bone collars are induced in the perichondrium adjacent to a cluster of ectopic hypertrophic chondrocytes (brackets). Despite the presence of clusters of ectopic hypertrophic chondrocytes, no ectopic bone collar is induced in the $/ h h^{-/-} ; P P R^{-/-} /$wild-type chimeric growth plate (c and d). Arrowheads denote where the eutopic prehypertrophic layer starts. Calcification inside the growth plate (d, asterisk) is ectopic cartilaginous mineralization caused by mutant hypertrophic chondrocytes. (e-g) In situ hybridization of sections of the anterior portion of the ribs from newborn mice with a mouse type $X$ collagen antisense probe. This portion of the wild-type ribs con-
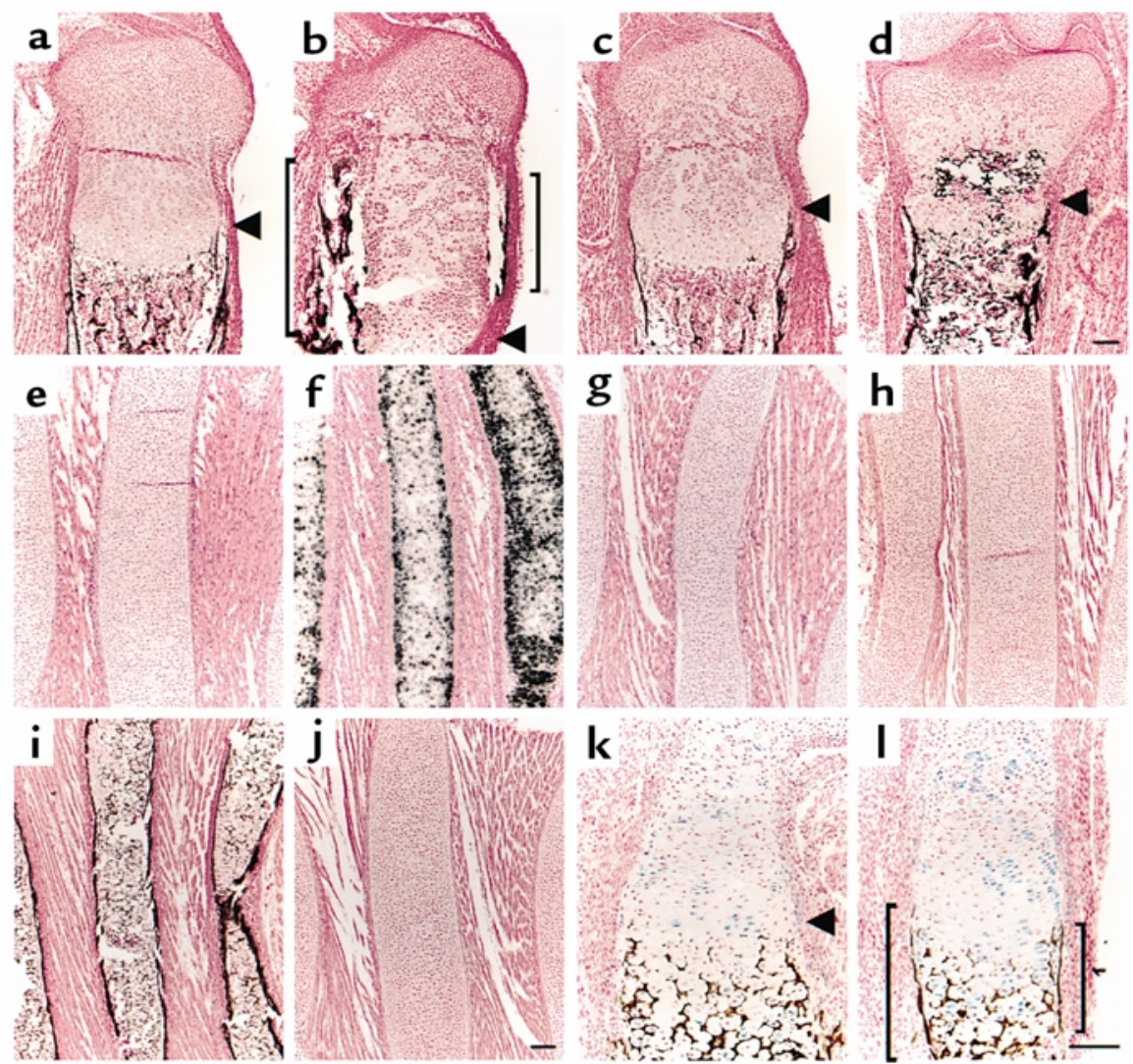

sists of proliferating chondrocytes (e), whereas chondrocytes in the $P T H r P^{-1-}$ ribs ectopically hypertrophy and express type X collagen mRNA (f). Introduction of a constitutively active $P P R$ transgene driven by the type ll collagen promoter (caPPR) reverses this ectopic hypertrophy (g). $(\mathbf{h}-\mathbf{j})$ Sections of the anterior portion of the ribs from newborn mice were stained by von Kossa method and counterstained with Nuclear Fast Red. Mineral is stained black; nuclei are stained red. The wild-type ribs have no bone collars (h), whereas the $P T H r P^{-1-}$ ribs have bone collars in association with the presence of ectopic hypertrophic chondrocytes (i). The PTHrP ${ }^{-1-}$;caPPR ribs, however, do not have bone collars, in association with the disappearance of ectopic hypertrophic chondrocytes (j). ( $\mathbf{k}$ and I) Sections of the humeri from d17.5 Ihh ${ }^{-/-} /$wild- $^{-}$ type chimera embryos were stained for $\beta$-galactosidase activity as well as stained by von Kossa method and counterstained with Nuclear Fast Red. Wild-type cells are stained blue; mineral is stained black; nuclei are stained red. In these mice, bone collars do not form in the perichondrium adjacent to clusters of $/ h^{-/-}$prehypertrophic/hypertrophic chondrocytes (arrowhead denotes where the prehypertrophic layer starts), whereas bone collars do form in the perichondrium adjacent to clusters of wild-type prehypertrophic/hypertrophic chondrocytes (brackets). Horizontal bar $=100 \mu \mathrm{m}$. 


\section{Figure 5}

Expression of osteoblastic markers in the chimeric growth plates. (a-c) In situ hybridization of sections of the tibiae from d17.5 embryos with a mouse osteopontin antisense probe. In the wild-type growth plate (a), osteopontin mRNA is expressed in osteoblasts as well as in mature chondrocytes, and its expression pattern overlaps the locations of bone collars and cartilaginous mineralization. In the $P P R^{-/-} /$wild-type chimera growth plate (b), there is ectopic expression of osteopontin mRNA in ectopic bone collars (bracket). Ectopic hypertrophic chondrocytes also express osteopontin mRNA (asterisk). In contrast, in the $I h h^{-/-} ; P T H / P P R^{-/-} /$wild-type chimera (c), there is no ectopic expression of osteopontin mRNA in the perichondrium, whereas ectopic hypertrophic chondrocytes still express osteopontin mRNA (asterisk). Arrowheads denote where the eutopic prehypertrophic layer starts. (d-f) In situ hybridization of sections of the tibiae from d17.5 embryos with a mouse osteocalcin antisense probe. In the wild-type growth plate (d), osteocalcin mRNA is expressed in mature osteoblasts. In the $P P R^{-/-} /$wild-type chimera (e), there is ectopic expression of osteocalcin mRNA in ectopic bone collars (bracket), whereas in the $I h h^{-/-} ; P P R^{-1-} /$ wild-type chimera (f), there is no ectopic expression of osteocalcin mRNA in the perichondrium. Arrowheads denote where the eutopic prehypertrophic layer starts. Horizontal bar $=100 \mu \mathrm{m}$.
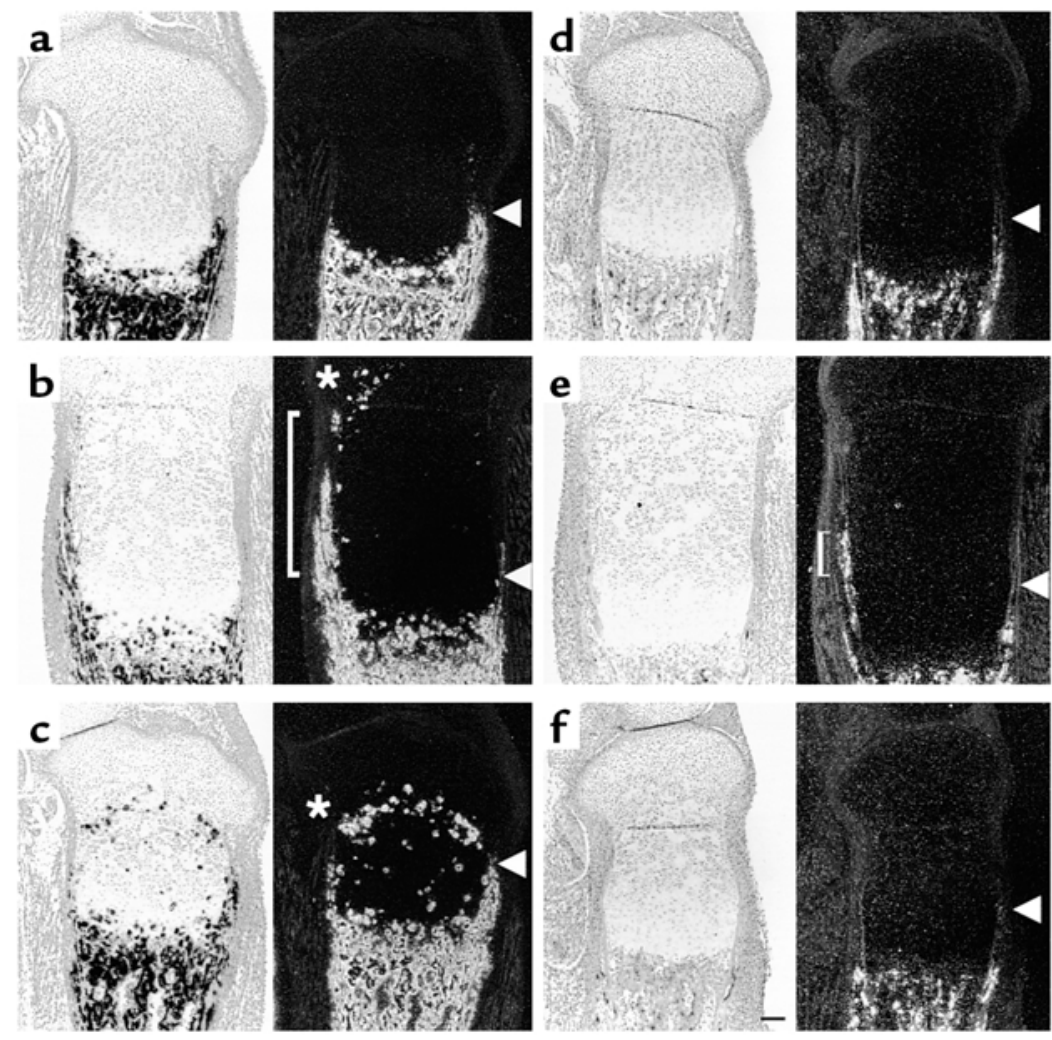

ed from wild-type cells. In these mice, bone collars do not form in the perichondrium adjacent to clusters of $\mathrm{Ihb}^{-1-}$ prehypertrophic/hypertrophic chondrocytes (Figure 4k), whereas bone collars do form in the perichondrium adjacent to clusters of wild-type prehypertrophic/hypertrophic chondrocytes (Figure 4l, brackets). These findings provide in vivo evidence that Ihh, secreted by prehypertrophic and hypertrophic chondrocytes, is locally required for induction of bone collar formation.

Bmp signaling alone is not sufficient for induction of bone collarformation. Because decapentaplegic (Dpp), a homolog of $B m p s$, works downstream of $\mathrm{Hb}$ signaling in Drosophila in some settings (32), and Bmps have been implicated in bone formation (33), it is possible that ectopic Ihh action in the growth plate of $\mathrm{PPR}^{-1-} /$ wild-type chimeric mice may work through $B m p$ signaling. Expression patterns of Bmp2, 4, 5, 6, and $7 \mathrm{mRNAs}$ were analyzed by in situ hybridization. In the growth plate of $P P R^{-1-} /$ wild-type chimeric mice, expression patterns of Bmp 4, 5, and 7 mRNAs do not change significantly, compared with those of wild-type mice (data not shown), whereas ectopic hypertrophic chondrocytes express Bmp2 and Bmp6 mRNAs (Figure 6, b and e, respectively), just as wild-type hypertrophic chondrocytes do (Figure 6, a and d, respectively). In the growth plate of $I b h^{-/-} ; P P R^{-/-} /$wild-type chimeric mice, ectopic hypertrophic chondrocytes also express Bmp2 and Bmp6 mRNAs (Figure 6, c and f, respectively), but they do not induce ectopic bone collar formation in the adjacent perichondrium. There is no significant difference in mRNA expression patterns of Bmp inhibitors such as Noggin, Gremlin, Dan, Chordin, and Follistatin between the growth plates of $I h b^{-/} ; P P R^{-1-} /$ wild- type chimera and $P P R^{-/} /$wild-type chimera mice (data not shown). Further, mRNA expression of Id1, one of the transcriptional target genes of $B m p$ signaling, is similarly upregulated in the growth plate of both chimeras (data not shown). These data suggest that $B m p$ signaling does not mediate the actions of Ihh on the periarticular growth plate. They also suggest that Bmp signaling alone cannot account for induction of bone collar formation in the perichondrium by adjacent prehypertrophic/hypertrophic chondrocytes.

\section{Discussion}

Ihb couples chondrogenesis and osteogenesis in endochondral bone development. Previous data suggested that Ihh and PTHrP participate in a negative feedback loop that regulates the rate of chondrocyte differentiation. However, the model was based on the experiments involving supraphysiological amounts of PTHrP and Ihh. The current study uses a chimeric system to demonstrate the role of Ihh in chondrogenesis in a more physiological setting. By comparing chimeric growth plates that differ genetically only by the presence or absence of the Ihb gene, we define the mechanism used by ectopic prehypertrophic/hypertrophic cells to extend the columns of normal proliferating chondrocytes. In the $P P R^{-/-} /$wild-type chimeric growth plates, but not in the $\mathrm{Ihb}^{-1-} ; \mathrm{PPR}^{-/} /$wildtype chimeric growth plates, ectopic Ihh regulates the amount of PTHrP synthesized in the periarticular region. When Ihh is synthesized nearer to the articular end of the growth plate, PTHrP synthesis is increased. In this way, Ihh signals the relative position of prehypertrophic and hypertrophic chondrocytes to the periartic- 


\section{Figure 6}

Bmp signaling alone is not sufficient for induction of bone collars. (a-c) In situ hybridization of sections of the tibiae from d17.5 embryos with a mouse $B m p 2$ antisense probe. In the wild-type growth plate (a), Bmp2 mRNA is expressed in hypertrophic chondrocytes and the perichondrium, whereas both in the $P P R^{-/-} /$wild-type chimeric growth plate (b) and in the Ihh ${ }^{-/-} ; P P R^{-/-} /$wild-type chimeric growth plate (c), ectopic hypertrophic mutant cells also express Bmp2 mRNA (arrowheads). (d-f) In situ hybridization of sections of the tibiae from $\mathrm{d} 17.5$ embryos with a mouse Bmp6 antisense probe. In the wild-type growth plate (d), Bmp6 mRNA is expressed in hypertrophic chondrocytes, whereas both in the $P P R^{-/-} /$wild-type chimeric growth plate (e) and in the $/ h^{-/-} ; P P R^{-/-} /$wild-type chimeric growth plate $(\mathbf{f})$, ectopic hypertrophic mutant cells also express Bmp6 mRNA (arrowheads). Horizontal bar $=100 \mu \mathrm{m}$.
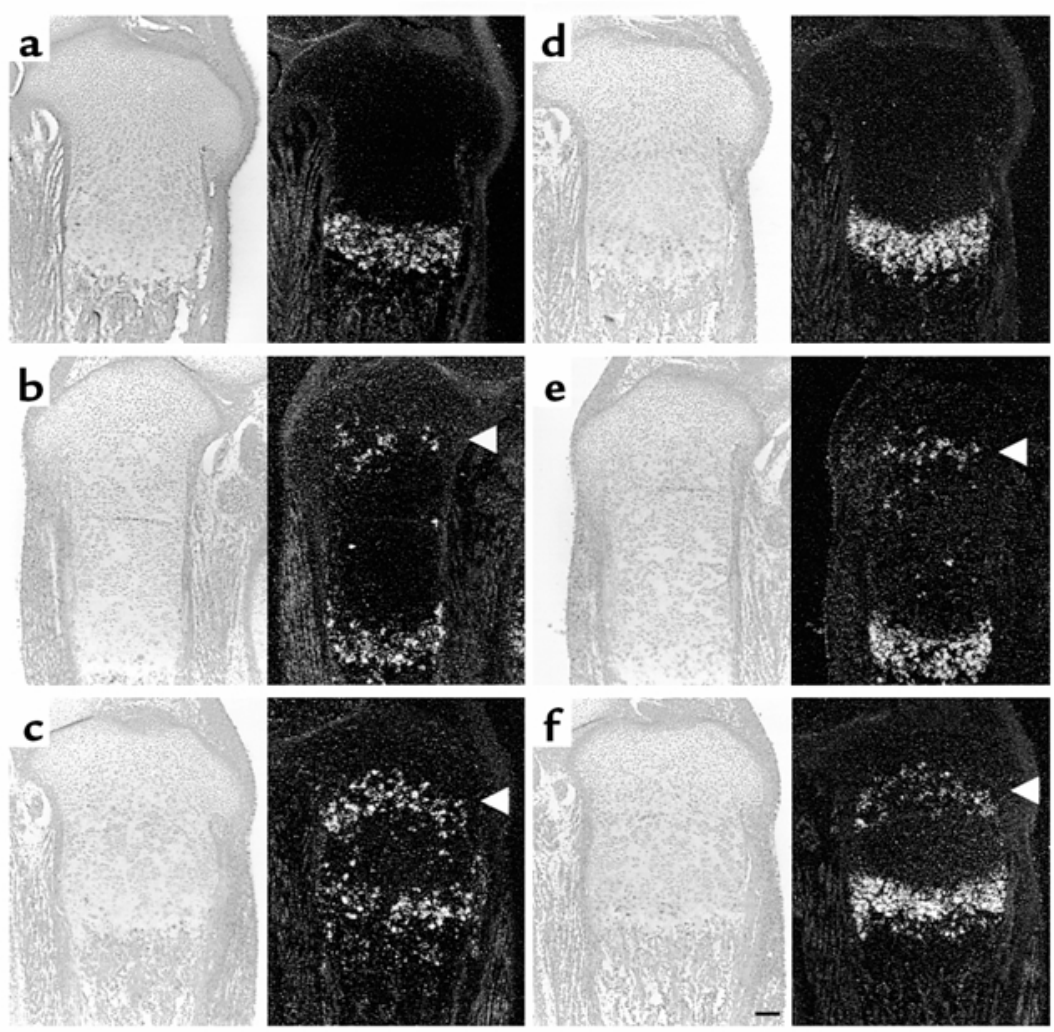

ular growth plate. PTHrP then delays the transition from proliferation to hypertrophy of growth plate chondrocytes. Thus, through PTHrP, Ihh controls the site at which hypertrophy occurs (Figure 7).

Ihh also determines the site of formation of the bone collar, which will become cortical bone. Previous work showed that $\mathrm{Ihb}^{-/-}$mice had no bone collar and that overexpression of Ihh by retroviral infection induced bone collar formation. However, it was unclear whether Ihh is locally required for this process in vivo, as the growth plates of $I h^{-1-}$ mice had a dramatically altered structure, and overexpression by retroviral infection involved supraphysiological amounts of Ihh. The current study shows that Ihh synthesized by prehypertrophic and hypertrophic chondrocytes is locally required for the induction of mature osteoblasts in the adjacent perichondrium in the growth plate with relatively normal architecture. The current study also shows that Bmp2 and Bmp6 synthesized by the same cells are not sufficient for this induction. Our in vivo data suggest that Ihh may induce differentiation of mature osteoblasts in the adjacent perichondrium, perhaps in synergy with Bmp2 and Bmp6 (Figure 7). It is noteworthy that Ihh converts only a select group of cells into mature osteoblasts. Perichondrial cells that will eventually become mature osteoblasts upon exposure to Ihh are a distinct population of cells, in that they express low levels of alkaline phosphatase, type I collagen and Cbfa1 mRNAs (4) (data not shown). Therefore, they are probably already committed to some extent toward the osteoblastic lineage and probably are susceptible to the induction of full maturation

\section{Figure 7}

Interactions of Ihh and PTHrP signaling pathways in the fetal growth plate. This figure shows a schema of the fetal growth plate of the mouse long bone. The end of the bone is at the top. As proliferating chondrocytes differentiate into prehypertrophic chondrocytes and then hypertrophic chondrocytes, they express Ihh. Ihh directly or indirectly stimulates PTHrP synthesis in the periarticular growth plate including the perichondrium and periarticular proliferating chondrocytes (long solid arrow). In this way, Ihh signals the relative position of prehypertrophic and hypertrophic chondrocytes to the periarticular growth plate. Ihh also has a positive effect on chondrocyte proliferation (short solid arrow). PTHrP then acts directly on columnar proliferating chondrocytes to delay their differentiation into prehypertrophic and hypertrophic chondrocytes, which synthesize Ihh (solid t-bar). Thus, through PTHrP, Ihh controls the site at which hypertrophy occurs. Ihh also acts on perichondrial cells to induce mature osteoblasts, which form a bone collar (white arrow).

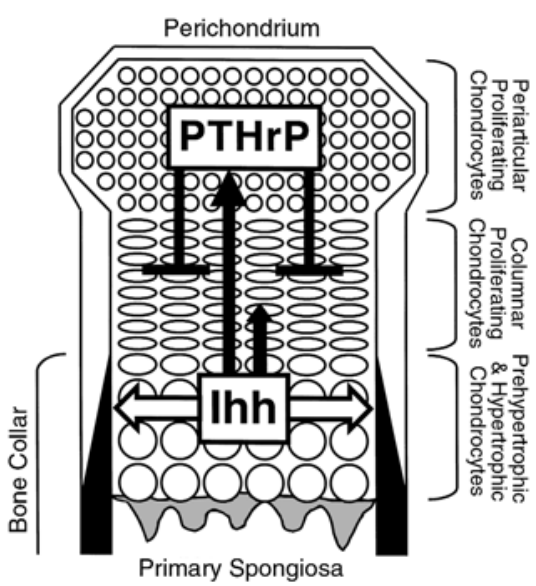


by $\mathrm{H} h$ signaling. Further study is required to clarify what signaling pathway initially commits perichondrial cells to the osteoblast lineage.

Involvement of Hh signaling in other modes of bone development. Although Ihh is required for the differentiation of bone collar osteoblasts, the role of Ihh in the differentiation of osteoblasts in other settings is less certain. These settings include endochondral osteoblasts of the primary spongiosa, which lay down a bone matrix on top of a cartilaginous matrix, and osteoblasts of intramembranous bones, such as the bones of the vault of the skull. The primary spongiosa expresses Ptc $1 \mathrm{mRNA}$, which is a marker for $\mathrm{H} h$ signaling. The source of $\mathrm{Hh}$ protein for this signaling might be Ihh secreted by prehypertrophic and hypertrophic chondrocytes. Hh proteins usually do not travel very far owing to the sequestering action of Ptc1 in responding cells. However, because hypertrophic chondrocytes do not express Ptc1, Ihh may be able to reach the primary spongiosa. $\mathrm{Ihb}^{-/-}$mice do have vascular invasion in the center of mineralized cartilage of the humerus, although osteoblasts with their associated matrix are not clearly present. However, mRNAs for proteins such as type I collagen are detectable, suggesting that early osteoblasts may be present. The severe abnormality in cartilage development and neonatal lethality of $\mathrm{Ihb}^{-1-}$ mice make it difficult to use these mice to determine the possible role of $I b h$ in the primary spongiosa.

Intramembranous bones such as the parietal bone do form in $\mathrm{Ihb}^{-/-}$mice. Although they are not normal, they do contain osteoblasts capable of ossification, presumably through an Ihh-independent pathway. Either another $\mathrm{Hh}$ molecule (Shh or Dhh) is active in these bones or, alternatively, osteoblasts in these bones do not require $\mathrm{Hh}$ signaling. From this perspective, the role of Ihh in bone collar formation emphasizes a special situation in which chondrocyte differentiation and osteoblast differentiation may need careful coordination. Ihh provides this coordination.

\section{Acknowledgments}

We thank E. Li for technical support in ES cell isolation, and the members of Endocrine Unit, Massachusetts General Hospital, for helpful discussion. This work was funded by a grant (to H. Kronenberg and A.P. McMahon) from NIH (P01 DK56246). U. Chung at the time of this research was a Medical Foundation and Charles A. King Trust Fellow.

1. Erlebacher, A., Filvaroff, E.H., Gitelman, S.E., and Derynck, R. 1995. Toward a molecular understanding of skeletal development. Cell. 80:371-378.

2. Marks, S.C., and Hermey, D.C. 1996. The structure and development of bone. In Principles of bone biology. J.P. Bilezikian, L.G. Raisz, and G.A. Rodan, editors. Academic Press. San Diego, California, USA. 3-14.

3. Chung, U.I., Lanske, B., Lee, K., Li, E., and Kronenberg, H. 1998. The parathyroid hormone/parathyroid hormone-related peptide receptor coordinates endochondral bone development by directly controlling chondrocyte differentiation. Proc. Natl. Acad. Sci. USA. 95:13030-13035.

4. Lee, K., Deeds, J.D., and Segre, G.V. 1995. Expression of parathyroid hormone-related peptide and its receptor messenger ribonucleic acids during fetal development of rats. Endocrinology. 136:453-463.

5. Karaplis, A.C., et al. 1994. Lethal skeletal dysplasia from targeted disruption of the parathyroid hormone-related peptide gene. Genes Dev. 8:277-289.

6. Lanske, B., et al. 1996. PTH/PTHrP receptor in early development and Indian hedgehog-regulated bone growth. Science. 273:663-666.
7. Amizuka, N., Warshawsky, H., Henderson, J.E., Goltzman, D., and Karaplis, A.C. 1994. Parathyroid hormone-related peptide-depleted mice show abnormal epiphyseal cartilage development and altered endochondral bone formation. J. Cell Biol. 126:1611-1623.

8. Jobert, A.S., et al. 1998. Absence of functional receptors for parathyroid hormone and parathyroid hormone-related peptide in Blomstrand chondrodysplasia. J. Clin. Invest. 102:34-40.

9. Zhang, P., Jobert, A.S., Couvineau, A., and Silve, C. 1998. A homozygous inactivating mutation in the parathyroid hormone/parathyroid hormone-related peptide receptor causing Blomstrand chondrodysplasia. J. Clin. Endocrinol. Metab. 83:3365-3368.

10. Karaplis, A.C., et al. 1998. Inactivating mutation in the human parathyroid hormone receptor type 1 gene in Blomstrand chondrodysplasia. Endocrinology. 139:5255-5258.

11. Karperien, M., et al. 1999. A frame-shift mutation in the type I parathyroid hormone (PTH)/PTH- related peptide receptor causing Blomstrand lethal osteochondrodysplasia. J. Clin. Endocrinol. Metab. 84:3713-3720.

12. Weir, E.C., et al. 1996. Targeted overexpression of parathyroid hormonerelated peptide in chondrocytes causes chondrodysplasia and delayed endochondral bone formation. Proc. Natl. Acad. Sci. USA. 93:10240-10245.

13. Schipani, E., et al. 1997. Targeted expression of constitutively active receptors for parathyroid hormone and parathyroid hormone-related peptide delays endochondral bone formation and rescues mice that lack parathyroid hormone-related peptide. Proc. Natl. Acad. Sci. USA. 94:13689-13694.

14. Schipani, E., Kruse, K., and Juppner, H. 1995. A constitutively active mutant PTH-PTHrP receptor in Jansen-type metaphyseal chondrodysplasia. Science. 268:98-100.

15. Krauss, S., Concordet, J.P., and Ingham, P.W. 1993. A functionally conserved homolog of the Drosophila segment polarity gene hh is expressed in tissues with polarizing activity in zebrafish embryos. Cell. 75:1431-1444.

16. Riddle, R.D., Johnson, R.L., Laufer, E., and Tabin, C. 1993. Sonic hedgehog mediates the polarizing activity of the ZPA. Cell. 75:1401-1416.

17. Echelard, Y., et al. 1993. Sonic hedgehog, a member of a family of putative signaling molecules, is implicated in the regulation of CNS polarity. Cell. 75:1417-1430.

18. Bitgood, M.J., and McMahon, A.P. 1995. Hedgehog and Bmp genes are coexpressed at many diverse sites of cell-cell interaction in the mouse embryo. Dev. Biol. 172:126-138.

19. Vortkamp, A., et al. 1996. Regulation of rate of cartilage differentiation by Indian hedgehog and PTH-related protein. Science. 273:613-622.

20. St-Jacques, B., Hammerschmidt, M., and McMahon, A.P. 1999. Indian hedgehog signaling regulates proliferation and differentiation of chondrocytes and is essential for bone formation. Genes Dev. 13:2072-2086.

21. Karp, S.J., et al. 2000. Indian hedgehog coordinates endochondral bone growth and morphogenesis via parathyroid hormone related-proteindependent and -independent pathways. Development. 127:543-548.

22. Kinto, N., et al. 1997. Fibroblasts expressing Sonic hedgehog induce osteoblast differentiation and ectopic bone formation. FEBS Lett. 404:319-323.

23. Nakamura, T., et al. 1997. Induction of osteogenic differentiation by hedgehog proteins. Biochem. Biophys. Res. Commun. 237:465-469.

24. Robertson, E.J. 1987. Embryo-derived stem cell lines. In Teratocarcinoma and embryonic stem cells. E.J. Robertson, editor. IRL Press. Oxford, United Kingdom. 71-112.

25. Bradley, A. 1987. Production and analysis of chimaeric mice. In Teratocarcinoma and embryonic stem cells. E.J. Robertson, editor. IRL Press. Oxford, United Kingdom. 113-152.

26. Zambrowicz, B.P., et al. 1997. Disruption of overlapping transcripts in the ROSA beta geo 26 gene trap strain leads to widespread expression of betagalactosidase in mouse embryos and hematopoietic cells. Proc. Natl. Acad. Sci. USA. 94:3789-3794

27. Rossert, J., Eberspaecher, H., and de Crombrugghe, B. 1995. Separate cis-acting DNA elements of the mouse pro-alpha 1(I) collagen promoter direct expression of reporter genes to different type I collagen-producing cells in transgenic mice. J. Cell Biol. 129:1421-1432.

28. Schipani, E., et al. 1993. Identical complementary deoxyribonucleic acids encode a human renal and bone parathyroid hormone (PTH)/PTH-related peptide receptor. Endocrinology. 132:2157-2165.

29. Stone, D.M., et al. 1996. The tumour-suppressor gene patched encodes a candidate receptor for Sonic hedgehog. Nature. 384:129-134.

30. Marigo, V., Davey, R.A., Zuo, Y., Cunningham, J.M., and Tabin, C.J. 1996. Biochemical evidence that patched is the Hedgehog receptor. Nature. 384:176-179.

31. Goodrich, L.V., Johnson, R.L., Milenkovic, L., McMahon, J.A., and Scott, M.P. 1996. Conservation of the hedgehog/patched signaling pathway from flies to mice: induction of a mouse patched gene by Hedgehog. Genes Dev. 10:301-312.

32. Ingham, P.W. 1998. Transducing hedgehog: the story so far. EMBO J. 17:3505-3511

33. Wozney, J.M., et al. 1988. Novel regulators of bone formation: molecular clones and activities. Science. 242:1528-1534. 